\title{
Development of CdZnTe doped with Bi for gamma radiation detection
}

\author{
V. Carcelén, ${ }^{* a}$ J. Rodríguez-Fernández, ${ }^{a}$ N. Vijayan, ${ }^{a b}$ P. Hidalgo,${ }^{c}$ J. Piqueras, ${ }^{c}$ N. V. Sochinskii, ${ }^{d}$ J. M. Perez ${ }^{e}$ \\ and E. Diéguez ${ }^{a}$
}

\author{
Received 23rd March 2009, Accepted 14th September 2009 \\ First published as an Advance Article on the web 28th September 2009 \\ DOI: $10.1039 / b 905810 a$
}

\begin{abstract}
Bulk CZT crystals doped with $\mathrm{Bi}\left(1 \times 10^{19}\right.$ at $\left./ \mathrm{cm}^{3}\right)$ have been grown by the Oscillatory Bridgman method, the growth velocity and the zinc concentration profile being improved by the insertion of a $\mathrm{Pt}$ tube acting as a cold finger. The stoichiometric uniformity was examined by energy dispersive X-ray analysis, and the zinc concentration was confirmed by inductively coupled plasma mass spectroscopy and cathodoluminescence measurements. The resistivity value was in the range of $8 \times 10^{8} \Omega \mathrm{cm}$, being smaller for the passivated sample, which at the same time had counter device properties.
\end{abstract}

\section{Introduction}

II-VI Semiconductor compounds based on cadmium zinc telluride (CZT) are getting increasing attention in the area of room temperature semiconductor detectors (RTSD). In fact, the huge number of applications such as X-ray and gamma ray detectors, ${ }^{1}$ e.g. in medical imaging applications, security devices, etc. ${ }^{2}$ are based on its physical and chemical properties like ${ }^{3}$ band gap above $1.5 \mathrm{eV}$, high atomic number of the constituent elements, high density, mechanical strength, the ability to grow high resistivity crystals ( $>10^{9} \Omega \mathrm{cm}$ ), photosensitivity, relatively good charge transport properties, relative good crystalline quality, etc.

Beside this, considering the whole process from the growth to the device application, there are important steps which must be taken into account because they can modify the material properties and in consequence the materials are used as radiation sensors. One of these steps is the surface passivation process which notably affects the device performance. Surface passivation must be carefully controlled, as it has been published in recent works. ${ }^{4}$ At the same time, the importance of a large number of defects which decreases the detector efficiency: grain boundary, twins, dislocations, cadmium vacancies and $\mathrm{Te}$ precipitates must be considered. In this way, Te inclusions and precipitates resulting from the growth process could be responsible for the leakage current and distorting carrier transport in CZT devices. ${ }^{5}$

Both of these factors have been considered in this paper by analyzing the same CZT samples with different passivation steps. The Oscillatory Vertical Bridgman Method was modified with a platinum tube acting as a cold finger which has been used in order to improve the crystal quality of the samples. ${ }^{6}$ Two sets of CZT samples with $10^{19} \mathrm{at} / \mathrm{cm}^{3}$ of Bi has been studied, following

${ }^{a}$ Laboratorio de Crecimiento de Cristales, Dpto. Física de Materiales, Facultad de Ciencias. Univ. Autónoma de Madrid, 28049 Cantoblanco, Spain.E-mail: veronica.carcelen@uam.es; Fax: +34-914978579

${ }^{b}$ National Physical Laboratory, New Delhi, 110 012, India

'Dpto. Física de Materiales, Facultad de Ciencias Físicas, Univ. Complutense de Madrid, 28040 Madrid, Spain

${ }^{d}$ Instituto de Microelectrónica de Madrid, CNM-CSIC, Polo Tecnológico de Madrid, Tres Cantos, 28760 Madrid, Spain

${ }^{e}$ Laboratorio General de Electrónica y Automática, CIEMAT, Edificio 22, Avenida Complutense, 22, E-28040 Madrid, Spain previously published works ${ }^{7}$ where it was demonstrated that a given $\mathrm{Bi}$ concentration increases the resistivity of the material. After irradiating the samples with radioactive sources, only the passivated sample was demonstrated for counter radiation quality.

\section{Experimental}

Crystal growth, processing and detector fabrication

$\mathrm{Cd}_{1-\mathrm{x}} \mathrm{Zn}_{\mathrm{x}} \mathrm{Te}(\mathrm{x}=0.15)$ with excess of $\mathrm{Cd}$ and doped with Bi has been grown by the Oscillatory Vertical Bridgman technique. The quartz ampoule with an internal diameter of $27 \mathrm{~mm}$ was located above the platinum tube support which is of the same dimensions as the quartz ampoule. The Pt tube acts as a cold finger in order to modify the original temperature conditions and in consequence to enhance the crystalline quality of the ingot. ${ }^{6}$

Before the growth process, the ampoule was cleaned in the sequence of $\mathrm{HCl} / \mathrm{HNO}_{3}$ (3:1) during a $24 \mathrm{~h}$ period followed by $\mathrm{HF}_{\text {conc }}$ for $3 \mathrm{~h}$, and rinsed with ethanol solvents, followed by a graphitization step carried out with $400 \mu \mathrm{l}$ of acetone at $1000{ }^{\circ} \mathrm{C}$. Chemicals of $\mathrm{Cd}, \mathrm{Zn}, \mathrm{Te}, \mathrm{Bi}$ elements $(6 \mathrm{~N}$, PURE METAL) were used as starting materials and an excess of $\mathrm{Cd}$ was used in the initial starting melt. The initial Bi concentration in the melt was $1 \times 10^{19} \mathrm{~atm} / \mathrm{cm}^{3}$. The growth process was carried out at a rate of $0.4 \mathrm{~mm} / \mathrm{h}$ with a temperature gradient in the solid liquid interface of $4.5^{\circ} \mathrm{C} / \mathrm{cm}$, followed by a cooling velocity of $5{ }^{\circ} \mathrm{C} / \mathrm{h}$ until it attains the rt (room temperature). As a result, an ingot of $27 \mathrm{~mm}$ in diameter and $90 \mathrm{~mm}$ in length was obtained, from which several wafers of 3-4 mm in thickness were cut at different positions along the axis of the ingot, the tip of the ampoule considered as the $0 \mathrm{~mm}$ position. For the analysis of this work, and considering the same $\mathrm{Zn}$ concentration along the ingot, two sets of single crystals were selected from the same position: set 1 with dimensions of $11 \times 8.8 \mathrm{~mm}^{2}$ and set 2 with dimensions of $9.2 \times 6.1 \mathrm{~mm}^{2}$, both with thickness of about $2 \mathrm{~mm}$. Both sets of samples were selected due to the highest crystal quality and unique grain.

The samples were lapped and polished with alumina powders (with sequential grain size of 5, 1 and 0.05 micron) suspended in deionized water on Buehler Chemomet polishing cloths. As one example, a polished CZT sample is shown in Fig. 1a. An average 

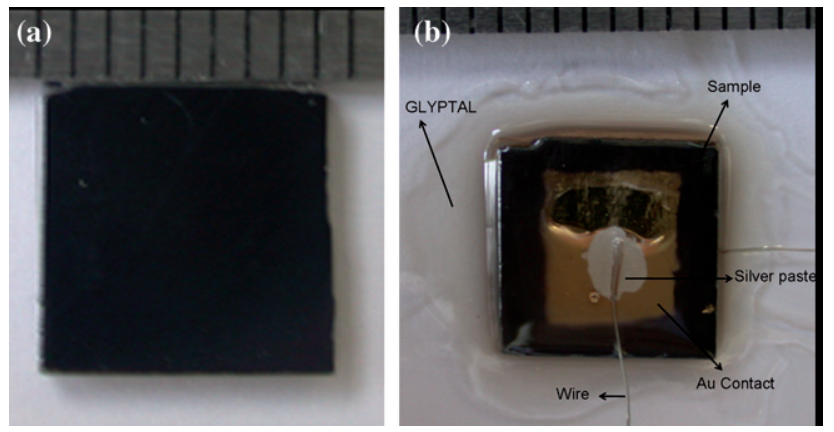

Fig. 1 One sample of set 1 after mechanical polishing (a), and after the device fabrication (b).

of $200 \mu \mathrm{m}$ of uneven material on both surfaces of the sample was polished away. Taking into account that the mechanical polishing causes the surface damage extending into the bulk crystal, the final surface must be submitted to a chemical etching carried with a $\mathrm{Br}_{2}$-methanol solution $(2 \%, 2 \mathrm{~min})$, then followed by rinsing the surface with methanol. Immediately after the chemical etching, gold ohmic contacts with an area of $6 \times 5 \mathrm{~mm}^{2}$ for SET1 and $4.6 \times 3.1 \mathrm{~mm}^{2}$ for the SET2 were deposited by vapor thermal deposition on both parallel faces.

Set of samples 1 was submitted to a surface passivation treatment by submerging the sample on ammonium fluoride $\left(\mathrm{NH}_{4} \mathrm{~F}\right) /$ deionized water/hydrogen peroxide $\left(\mathrm{H}_{2} \mathrm{O}_{2}\right)$ solution of $2.68 \mathrm{~g} / 17 \mathrm{ml} / 8 \mathrm{ml}$ for $10 \mathrm{~min}^{4}$, considering that this chemical solution showed the best passivation efficiency ${ }^{4}$ due to the fact that an original surface enriched with $\mathrm{Te}$ is fully oxidized to $\mathrm{TeO}_{2}$ after the procedure described above, with a consequence of a more stoichiometric surface and a minimum leakage current. On the contrary, the set of samples 2 was not passivated in order to compare the experimental results of both sets of CZT samples.

Mechanical contacts were deposited on both sets of samples by means of a silver paste and metallic electrodes which were deposited over the Au contact. Finally, both sets of samples were wrapped with commercial GLYPTAL lacquer which acted as an insulating boundary. Fig. 1b shows a photograph of one sample of set 1 .

Characterization. $\mathrm{Bi}$ and impurities concentrations were measured using inductively coupled plasma mass spectroscopy (ICP-MS) with a mass spectrometer ELAN-6000, PE-Sciex (U.S.A). The cathodoluminescence (CL) measurements, spectra and images, were carried out in a Leica 440 SEM and in a Hitachi $2500 \mathrm{SEM}$ at $20 \mathrm{kV}$ of electron beam energy in both cases and at a temperature of $78 \mathrm{~K}$, by using a R5509 Hamamatsu photomultiplier tube as signal detector. The EDX (energy dispersive Xray analysis) measurements were carried out in a Leica 440 SEM equipped with a Bruker AXS QUANTAX system. The homogeneity of the sample was studied by the etch-pit densities (EPD) with $\mathrm{H}_{2} \mathrm{O}: \mathrm{HNO}_{3}: \mathrm{HF}: \mathrm{AgNO}_{3(\mathrm{~S})}$ solution, and analyzed on a Hitachi S-3000N (SEM). Room temperature resistivity measurements were carried out using a Keithley electrometer (Model 6514) and ET NHQ 105L DC high voltage power supply.

The nuclear electronics chain used for testing the CZT detectors was based on an A250 charge sensitive preamplifier from Amptek, AC coupled to the detector top electrode. The detector cathode was biased by using a Bertan high voltage power supply. An Ortec 885 Gaussian shaper amplifier module was used to filter and shape the preamplifier output signal. The shaping time was set to $1.5 \mu$ s. The acquisition system was based on an MCA PC card from Aptec. The stability and resolution of the whole setup were checked before data acquisition by using a precision test pulse generation from Canberra (814FP) and a commercial $\mathrm{CdTe}$ detector from Acrorad. ${ }^{22} \mathrm{Na},{ }^{133} \mathrm{Ba}$ and ${ }^{241} \mathrm{Am}$ radioactive sources were used (see Fig. 2).

\section{Results and discussions}

The composition of both sets of samples is homogeneous as it has been measured by EDX which shows a uniform concentration of $\mathrm{Cd}, \mathrm{Zn}, \mathrm{Te}$ and $\mathrm{Bi}$ (49.25, 7.5, 42.75 and 0.7 at.\%, respectively), as was confirmed by CL for the near band gap emission following the well established Lennard-Jones expression $\mathrm{E}_{\mathrm{GAP}}=(1.606+$ $\left.0.322 \mathrm{x}+0.463 \mathrm{x}^{2}\right) \mathrm{eV} .{ }^{8}$ In addition to this, the $\mathrm{Zn}$ concentration on both sets of samples coincides with the composition along the whole axial direction of the ingot $(7.5 \pm 0.1$ at.\%) with the exception of the bottom and top regions of the ingot, as a consequence of the thermal environments on the solid/liquid interface created by the presence of the Pt holder acting as a cold finger. ${ }^{6}$

The cathodoluminescence studies demonstrate the good quality of both sets of samples (Fig. 3), showing one radiative

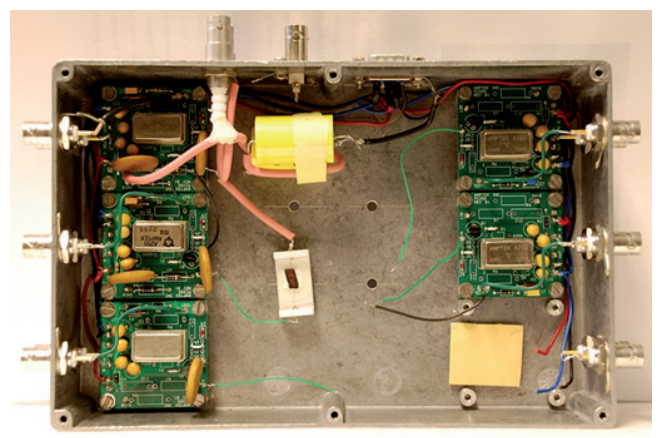

Fig. 2 Testing equipment: test box with an installed CdZnTe device.

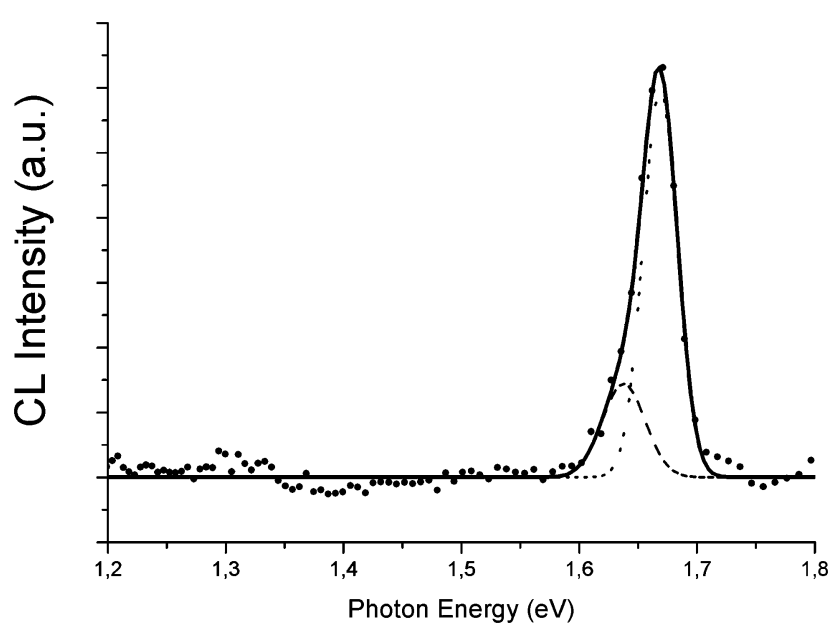

Fig. $3 \mathrm{CL}$ spectra of CdZnTe:Bi (high resistivity) at $78 \mathrm{~K}$. 
emission centered at $1.666 \mathrm{eV}$, which is attributed to the near band gap of CdZnTe in this case of $\mathrm{x}=0.15$ for zinc, and the other peak at $1.635 \mathrm{eV}$ could be attributed to excitonic transitions (a more detailed analysis is necessary to establish this point). Other interesting results are the absence of the $1.4 \mathrm{eV}$ and $1.1 \mathrm{eV}$ bands, generally assigned to defects bands associated with cadmium vacancies and Te interstitial, respectively, which could indicate the decrease of $\mathrm{Cd}$ vacancies and therefore the excellent quality of the samples. In fact, these results could be the consequence of the $\mathrm{Cd}$ excess and at the same time the dopant effect of the amphoteric $\mathrm{Bi}$ atom in order to compensate for the $\mathrm{Cd}$ vacancies and Te in the interstitial position. ${ }^{7}$ However, the EPD analysis shows a value of $2-3 \times 10^{4} \mathrm{~cm}^{-2}$ for both sets of samples, as one can see in Fig. 4, which demonstrates the good crystalline quality of the studied samples.

In order to analyze the device properties of both samples, the detectors fabricated by the steps described in the Experimental section were studied electrically. The resistivity measurement was carried out on both sets of samples (see Table 1), and the I-V results are shown in Fig. 5 and 6 for both samples in the voltage range of $\pm 500 \mathrm{~V}$. The resistivity obtained is $8 \times 10^{8} \Omega \mathrm{m}$ for one sample of set 1 (passivated), and $2 \times 10^{9} \Omega \mathrm{m}$ for one sample of set 2 (non passivated). These values are optimal for applications like sensor radiation, although the cadmium vacancies are important defects that decrease the resistivity of the crystals, but in this work, the $\mathrm{Cd}$ excess and bismuth dopant were used to compensate the for cadmium vacancies.

In order to know the device properties of both samples, radiation measurements were carried out with ${ }^{22} \mathrm{Na},{ }^{133} \mathrm{Ba}$ and ${ }^{241} \mathrm{Am}$ radioactive sources. The detector was biased at $300 \mathrm{~V}$ during the

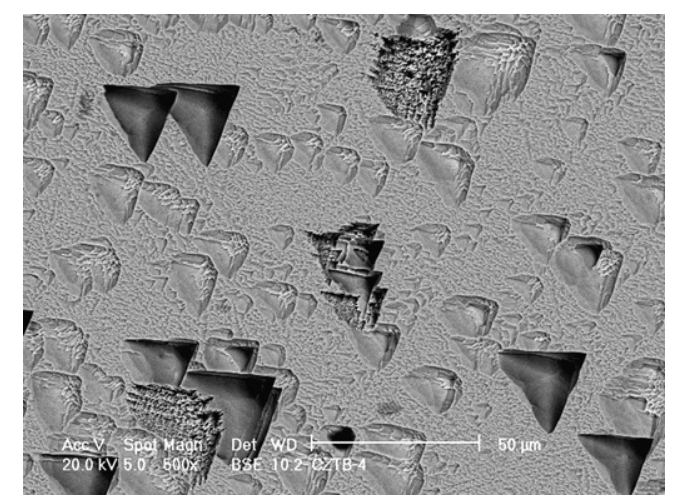

Fig. 4 SEM picture of etch pits on a CdZnTe:Bi (1) crystal of set 1.

Table 1 Resistivity values for both sets of samples

\begin{tabular}{lll}
\hline Set & Number of sample & Resistivity $/ \Omega \mathrm{cm}$ \\
\hline 1 & 1 & $7.5 \times 10^{8}$ \\
1 & 2 & $8.3 \times 10^{8}$ \\
1 & 3 & $7.7 \times 10^{8}$ \\
1 & 4 & $8.0 \times 10^{8}$ \\
1 & 5 & $8.2 \times 10^{8}$ \\
2 & 1 & $1.5 \times 10^{9}$ \\
2 & 2 & $2.0 \times 10^{9}$ \\
2 & 3 & $1.7 \times 10^{9}$ \\
2 & 4 & $2.2 \times 10^{9}$ \\
2 & 5 & $2.5 \times 10^{9}$ \\
\hline
\end{tabular}

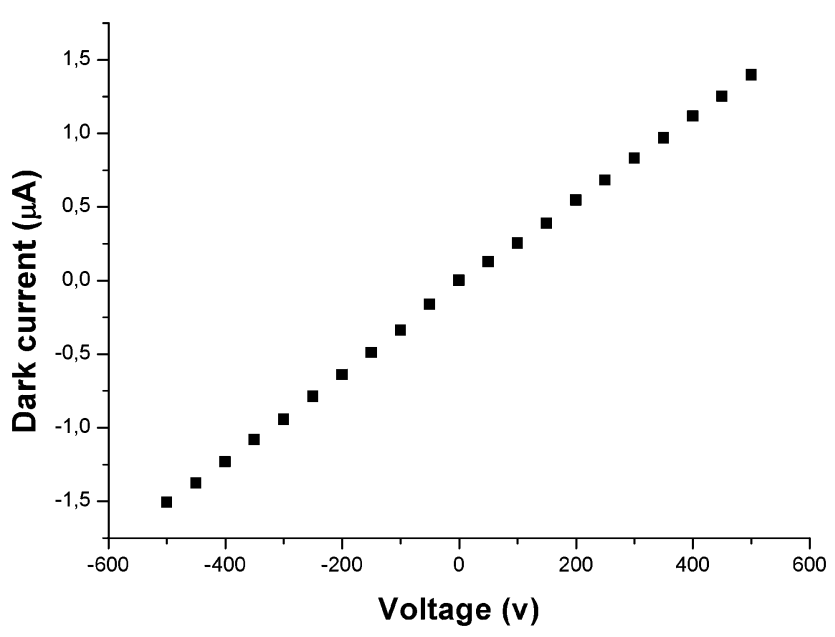

Fig. 5 A representative I-V curve for CdZnTe:Bi (1) sample of set 1.

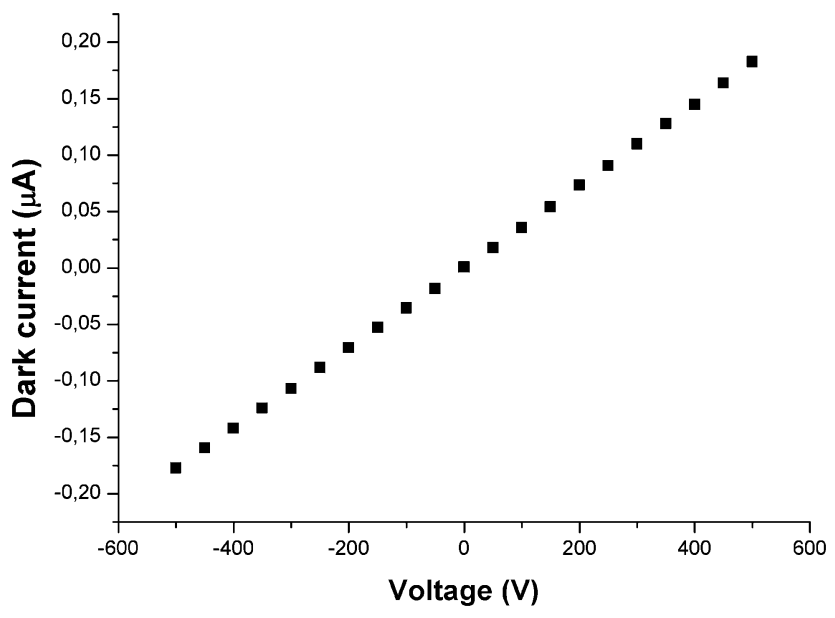

Fig. 6 A representative I-V curve for CdZnTe:Bi (2) sample of set 2.

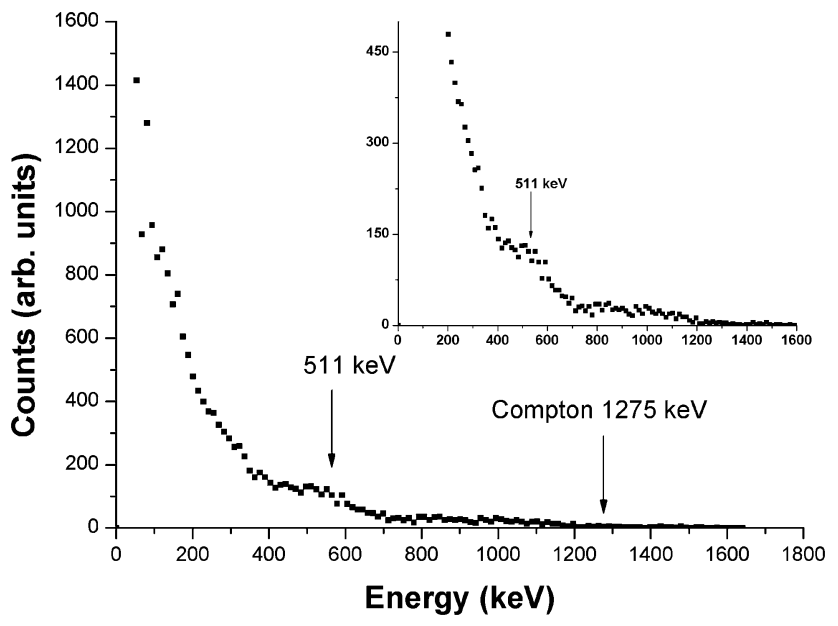

Fig. 7 Room temperature spectra acquired by irradiating a CdZnTe:Bi(1) crystal (300 V bias) of set 1 with a ${ }^{22} \mathrm{Na}$ source.

acquisitions. The three sources were detected, thus demonstrating detection capabilities from $60 \mathrm{keV}\left({ }^{241} \mathrm{Am}\right)$ up to 1.3 $\mathrm{MeV}\left({ }^{22} \mathrm{Na}\right)$. Nonetheless, the detector showed poor 


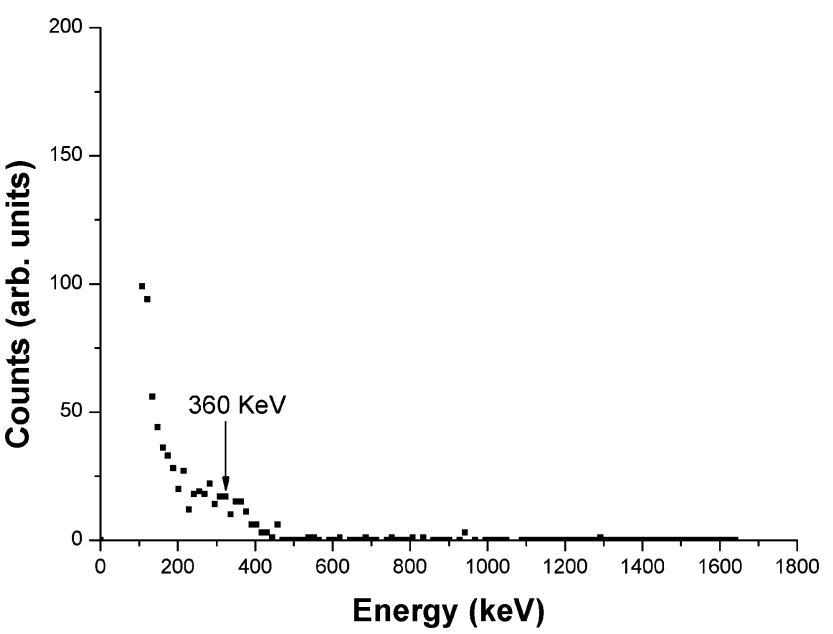

Fig. 8 Room temperature spectra acquired by irradiating a CdZnTe:Bi(1) crystal (300 V bias) of set 1 with a ${ }^{133} \mathrm{Ba}$ source.

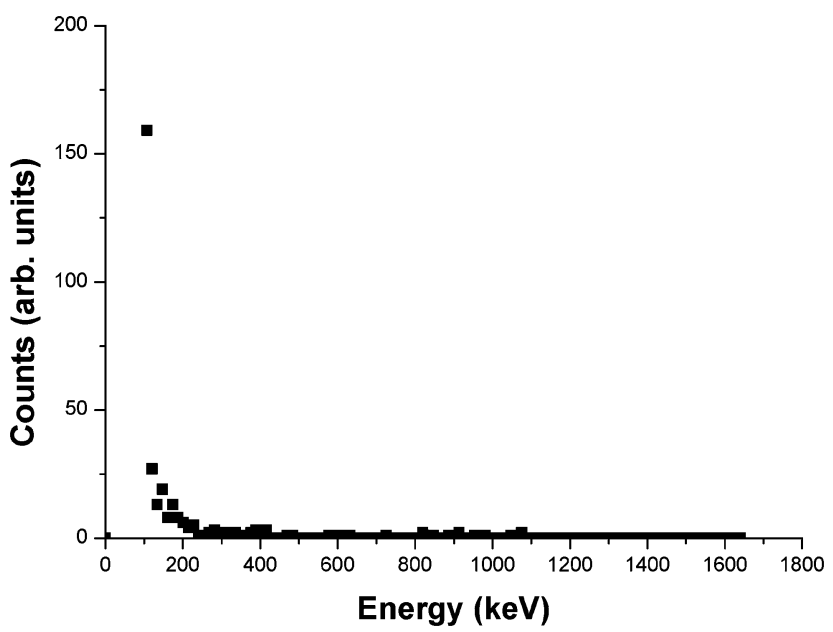

Fig. 9 Room temperature spectra without source of a CdZnTe:Bi(1) crystal (300 V bias) of set 1.

spectroscopic capabilities. As an example, in Fig. 7 is presented a spectrum acquired for a sample of set 1 with a $10 \mu \mathrm{Ci}{ }^{22} \mathrm{Na}$ radioactive source located at $\sim 5 \mathrm{~cm}$ on top of the detector cathode for $600 \mathrm{~s}$. The $511 \mathrm{keV}$ photopeak is coarsely evidenced, whereas the $1275 \mathrm{keV}$ line is only seen from its Compton edge. Another example is presented in Fig. 8 a spectrum with a ${ }^{133} \mathrm{Ba}$ radioactive source. The peak around $360 \mathrm{keV}$ is a $\mathrm{Ba}$ emission. Finally, in Fig. 9, we have shown the spectrum acquired without a radioactive source.

The same experiment which has been carried out with samples in set 2 shows reduced behavior which might be due to the lack of passivation process.

The high leakage current measured reveals a concentration of electrically active defects much higher than expected. This point is under study and methods for improving the detector quality are already under testing. Once we reach acceptable values of defects, we will concentrate on the detector homogeneity, a crucial property for the detector quality.

In summary, the passivation effect is not noticeable in both resistivity measurements but it is very important for the improvement of the counter application, which must be considered for future applications and studies.

\section{Conclusions}

CdZnTe:Bi bulk crystals with Cd excess have been grown by the Bridgman Method modified with a Pt tube acting as a cold finger and supporting the quartz ampoule. Two sets of single crystals were cut from the initial ingot, one of them submitted to a passivation process. Both sets of samples show low defect density and high crystalline quality, with resistivity values of $8 \times$ $10^{8} \Omega \mathrm{m}$ and $2 \times 10^{9} \Omega \mathrm{m}$. The passivated set of samples showed a low resistivity value and demonstrated counter radiation capability, showing the importance and interest in the passivation step.

\section{Acknowledgements}

This work was partially supported by the following projects: ESP2006-09935, Spanish "Ministerio de Educación y Ciencia"; S-0505/MAT-0279, Spanish "Comunidad de Madrid"; FP7SEC-2007-01, European Commission, and Contract number $14240 / 00 / \mathrm{NL} / \mathrm{SH}$, European Space Agency. V.C. is thankful to the Ministry of Education and Science, Spain for financial support. N.V. is grateful to Department of Science and Technology, Govt. of India for providing the BOYSCAST fellowship. J.R.F. is thankful to the Universidad Autónoma of Madrid for financial support.

\section{References}

1 (a) G. Yang, W. Jie, Q. Li, T. Wang, G. Q. Li and H. Hua, J. Cryst. Growth, 2005, 283, 431; (b) T. Wang, W. Jie, J. Zhang, G. Yang, D. Zeng, Y. Xu, S. Ma, H. Hua and K. He, J. Cryst. Growth, 2007, 304, 313; (c) M. Zha, A. Zappettini, D. Calestani, L. Marchini, L. Zanotti and C. Paorici, J. Cryst. Growth, 2008, 310, 2072; (d) I. Jung, H. Krawczynski, A. Burger, M. Guo and M. Groza, Astropart. Phys., 2007, 28, 397.

2 G. Sato, T. Takahashi, M. Sugiho, M. Kouda, T. Mitani, K. Nakazawa, Y. Okada and S. Watanabe, IEEE Trans. Nucl. Sci., 2002, 49, 1258.

3 T. E. Schlesinger, J. E. Toney, H. Yoon, E. Y. Lee, B. A. Brunett, L. Franks and R. B. James, Mater. Sci. Eng., R, 2001, 32, 103.

4 (a) Wang Xiaoqin, Jie Wanqi, Li Qiang and Gu Zhi, Mater. Sci. Semicond. Process., 2005, 8, 615; (b) Alireza Kargar, Andrew M. Jones, Walter J. McNeil, Mark J. Harrison and Douglas S. McGregor, Nucl. Instrum. Methods Phys. Res., Sect. A, 2006, 558, 497; (c) K. C. Mandal, S. H. Kang, M. Choi, A. Kargar, M. J. Harrison, D. S. McGregor, A. E. Bolotnikov, G. A. Carini, G. C. Camarda and R. B. James, IEEE Trans. Nucl. Sci., 2007, 54, 802.

5 G. A. Carini, A. E. Bolotnikov, G. S. Camarda, G. W. Wright, R. B. James and L. Li, Appl. Phys. Lett., 2006, 88, 143515.

6 (a) V. Carcelen, N. Vijayan, E. Dieguez, A. Zappettini, M. Zha, L. Sylla, A. Fauler and M. Fiederle, J. Optoelect. Adv. Mat., 2008, 10, 3135; (b) V. Carcelén, N. Vijayan, J. Rodriguez-Fernandez, P. Hidalgo, J. Piqueras, N. V. Sochinskii, J. M. Perez and E. Dieguez, J. Cryst. Growth, 2009, 311, 1264.

7 (a) E. Saucedo, O. Martinez, C. M. Ruiz, O. Vigil-Galán, I. Benito, L. Fornaro, N. V. Sochinskii and E. Diéguez, J. Cryst. Growth, 2006, 291, 416; (b) E. Saucedo, C. M. Ruiz, V. Bermúdez, E. Diéguez, E. Gombia, A. Zappettini, A. Baraldi and N. V. Sochinskii, J. Appl. Phys., 2006, 100, 104901.

8 (a) W. Stadler, D. M. Hofmann, H. C. Alt, T. Muschik, K. K. Meyer, E. Weigel, G. Müller-Vogt, M. Salk, E. Rupp and K. W. Benz, Phys. Rev. B: Condens. Matter Mater. Phys., 1995, 51, 10619; (b) J. L. Reno and E. D. Jones, Phys. Rev. B: Condens. Matter Mater. Phys., 1992, 45, 1440 . 